\title{
BMJ Open Diagnostic barriers for somatic symptom disorders in primary care: study protocol for a mixed methods study in Germany
}

Christina Heinbokel, ${ }^{1}$ Marco Lehmann, ${ }^{1}$ Nadine Janis Pohontsch, ${ }^{2}$ Thomas Zimmermann, ${ }^{2}$ Astrid Althaus, ${ }^{2,3}$ Martin Scherer, ${ }^{2}$ Bernd Löwe ${ }^{1}$

To cite: Heinbokel C, Lehmann M, Pohontsch NJ, et al. Diagnostic barriers for somatic symptom disorders in primary care: study protocol for a mixed methods study in Germany. BMJ Open 2017;7:e014157. doi:10.1136/ bmjopen-2016-014157

- Prepublication history for this paper is available online. To view these files please visit the journal online (http://dx.doi org/10.1136/bmjopen-2016014157).

Received 5 September 2016 Revised 17 May 2017 Accepted 21 June 2017

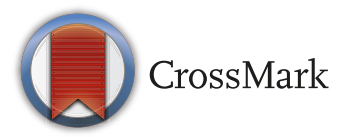

${ }^{1}$ Psychosomatic Medicine and Psychotherapy, University Medical Center HamburgEppendorf and Schön Clinic Hamburg Eilbek, Hamburg, Germany

${ }^{2}$ General Practice/Primary Care, Center for Psychosocial Medicine, University Medical Center Hamburg-Eppendorf, Hamburg, Germany

${ }^{3}$ Department of General

Medicine Cologne, University Hospital of Cologne, Cologne Germany

Correspondence to Dr Christina Heinbokel; c.heinbokel@uke.de

\section{ABSTRACT}

Introduction Somatoform or somatic symptom disorders $((\mathrm{S}) \mathrm{SD})$ are common and have a negative impact on the patients' health-related quality of life, healthcare use and costs. In primary care, which is central to the management of $(\mathrm{S}) \mathrm{SD}$, diagnosis and treatment tend to be delayed. There is a significant lack of evidence regarding the barriers in the diagnostic process of (S)SD in primary care and how interventions should be tailored to address them. The aim of this study is to analyse the diagnostic process in primary care that results in the diagnosis or nondiagnosis of a (S)SD.

Methods and analysis This mixed methods study will investigate the topic with qualitative methods, subsequently proceeding to a quantitative phase where the initial results will be validated and/or generalised. First, focus groups will explore meanings and patterns, inconsistencies and conflicts in general practitioners' (GPs) thoughts and behaviours when diagnosing (S)SD Second, the results of these focus groups will be used to develop interview guidelines for subsequent faceto-face interviews. Patients and their treating GPs will be interviewed separately on how they experience the history of illness, the diagnostic process and treatment. Third, based on the results of the first two study parts, a questionnaire will be derived and a nationwide survey among German GPs will be conducted, quantifying the barriers and difficulties identified before.

Ethics and dissemination Ethics approval was obtained from the Ethics Committee of the Hamburg Medical Association, Germany (approval number PV4763). The results of this study will be disseminated through conference presentation and publications in peer-reviewed journals.

Trial registration number The study is registered in the German Clinical Trial Register (DRKS), DRKS-ID DRKS00009736.

\section{INTRODUCTION}

Somatic symptoms are central to some mental disorders in the Diagnostic and Statistical Manual of Mental Disorders (DSM).${ }^{1}$ DSM-5's somatic symptom disorder (SSD) succeeds the DSM-IV concept of somatoform disorders
Strength and limitations of this study

- This study will provide methodologically wellfounded knowledge about the diagnostic process and management of somatoform or somatic symptom disorders ((S)SD) in primary care.

- Both Diagnostic and Statistical Manual of Mental Disorders (DSM)-IV and DSM-5 conceptualisations will be evaluated from GPs' perspectives.

- The study provides information on GPs' sociodemographic characteristics whose influence on the diagnostic process has not been investigated yet

- Healthcare providers other than GPs are not part of the study.

- Results might only apply for German healthcare system and its providers.

(SD). Psychopathological features such as disproportionate thoughts and feelings are an integral part of the new diagnosis of SSD whereas one core criterion is not included anymore, namely a medical condition cannot completely explain the severity of symptoms. ${ }^{12}$ The current ICD-10 diagnosis of SD is similar to the one in DSM-IV but will soon be replaced by an ICD-11 version, probably resembling DSM-5's SSD criteria. SSD was introduced in order to adjust the concept to the demand in primary care and to overcome body-mind dualism by no longer valuing the extent of explicability of the somatic symptoms. ${ }^{1}$ At large, SSD is assumed to merge patients formerly diagnosed with SD and those diagnosed with hypochondriasis who present somatic symptoms. ${ }^{1}$ However, most research until now refers to $\mathrm{SD}$ and data about the current concept of SSD are limited yet. Therefore, this study protocol will distinguish thoroughly between the concept of SD and SSD or refer to both concepts as 'somatoform or somatic symptom disorders ((S)SD)' 
in order to report precisely while taking the up-to-date diagnostic concept into account.

Primary care is central to the identification, management and treatment of SD. Given that patients with somatic complaints initially seek help in primary care settings but await specialised therapy on average for 6 years, ${ }^{34}$ it is of interest which diagnostic framework general practitioners (GPs) apply to these patients. Early detection and treatment of patients with SD is also important because these patients are at high risk for suicidal actions. ${ }^{5} 6$ However, there is a significant lack of evidence regarding the diagnostic process of SD in primary care and how interventions should be selected to address its barriers. ${ }^{7}$ The aforementioned conceptual shift further challenges this question.

The prevalence of SSD is estimated to be around $5 \%-7 \%$ in the general population. ${ }^{1}$ In the primary care setting, the prevalence of SD is reported within a wide range between $5 \%$ and $35 \% .^{8-11}$ In both inpatient and outpatient settings, SD are underdiagnosed..$^{12-16}$ The wide range in prevalence of SD in primary care settings indicates that diagnostic approaches, recognition or labels vary. ${ }^{15}$ The diagnostic process is further challenged by high comorbidity rates with other psychological disorders, in particular anxiety disorders and depression. ${ }^{11}{ }^{17-19} \mathrm{SD}$ are associated with significantly reduced health-related quality of life,${ }^{20}$ their impact being comparable to diseases with clear organic origins. ${ }^{21}$ Psychological interventions, for example, cognitive behavioural therapy, significantly improve symptoms and reduce healthcare costs. ${ }^{20}{ }^{22-26}$ If not diagnosed and treated properly, ongoing medical investigations and procedures might cause iatrogenic harm and become a financial burden for society. ${ }^{27-29}$ These findings underline the importance of an early identification and stratification of patients suffering from (S) SD.

In general, making the diagnosis of any condition consists of its identification from the patient's report of symptoms, knowing about its diagnostic criteria and recording it. ${ }^{15}$

So far, few interventions have focused on overcoming barriers in diagnosing (S)SD: until now, the training of GPs in the assessment and treatment of patients with SD has not been shown to have any relevant effect on clinical outcome. ${ }^{3031}$ Collaborative care approaches that include psychiatric consultations in primary care practices are promising. ${ }^{32} 33$

A review from parts of our study group has given insight into possible barriers in diagnosing SD. ${ }^{34}$ These barriers can be categorised as patient-related, doctor-related, interactional, situational and conceptual ones. For example, a preference for biomedical disease models is known to be a patient-related as well as doctor-related barrier. Limited time for a consultation is an example for a situational barrier. However, few of the included publications investigated GPs directly and identification of barriers to the diagnosis of (S) SD was mainly not the focus of the studies included. Furthermore, the review contains studies regarding various psychosomatic diagnoses and syndromes and, therefore, results cannot easily be transferred to isolated (S) SD diagnoses. Lastly, the barriers could not be prioritised regarding their clinical impact and ground on different levels of methodological quality. Therefore, the importance for primary care of any of the listed barriers could not be assessed. Some barriers such as the nature of symptoms or the socio-medico-legal context are unlikely to be overcome. It is important, however, that these barriers are recognised so that resources and support can be directed reasonably. Barriers regarding communication, attitudes, behaviour or education could be addressed in interventional studies or public health initiatives. To this end, this study will explore the GPs' specific experiences within the diagnostic process of $(\mathrm{S})$ SD.

\section{Aim}

The aim of this mixed methods study is to analyse the diagnostic process in primary care that results in the diagnosis or non-diagnosis of a (S) SD and to give a concise description of this process.

The objectives of this study are:

- Methodologically sound approval of yet known barriers and identification of yet unknown barriers involved in the process of diagnosing $(\mathrm{S}) \mathrm{SD}$ in primary care.

- Assessment of the relevance of the identified barriers.

- Development of recommendations to facilitate the diagnosis and management of (S)SD.

This study will provide insight into the GPs' views of their roles regarding patients with (S)SD-whether it is diagnosing, treating, guiding or keeping harm from these patients. It will be assessed in how far the conceptual shift from SD to SSD might influence the management of these patients. Moreover, the factors that hinder an early treatment of these patients will be explored.

\section{METHODS AND ANALYSIS \\ Study design}

A cross-sectional, qualitative-quantitative design as shown in figure 1 will be used to identify barriers. It begins with qualitative methods and then proceeds to a quantitative phase where the initial results will be tested or generalised. The project consists of four parts: (1) Focus groups will be used to explore meanings and patterns, inconsistencies and conflicts in GPs' thoughts and behaviours when diagnosing (S)SD. (2) The results of these focus groups will be used to develop interview guidelines for the subsequent face-to-face interviews. Patients and their treating GPs will be interviewed separately on how they experience the history of illness, its diagnostic process and its treatment. In this part, distinct diagnostic processes of (S) SD will be analysed. (3) The difficulties identified in the diagnostic process in primary care will be quantified by means of a nationwide survey among German GPs. This 


\section{Part I - II (qualitative) Focus groups Dyadic interviews}

\section{Part III (quantitative) Nationwide survey}

Figure 1 Study design.

questionnaire will also assess the acceptance of possible interventions. The study was started on 1 September 2015 and will be finished 31 July 2018.

Given that we are currently at the transition from the DSM-IV concept of SD to the DSM-5 concept of SSD, both diagnostic conceptualisations will be considered and diagnostic barriers and facilitators that are specific to each of them will be investigated. This study will be conducted in equal parts by the Institute for Psychosomatic Medicine and Psychotherapy and the Department of General Practice/Primary Care.

\section{Part I: Focus groups}

Focus groups provide insight into GPs' opinions, multifaceted motivations and behaviours within the diagnostic process of (S)SD. Six focus groups, lasting 2 hours each, will be conducted. The target population are GPs. Ten participants will be invited to each focus group to finally obtain a group of eight GPs. One pilot focus group will be conducted with GPs at the annual meeting of the German College of General Practitioners and Family Physicians. A random sample of 1000 GPs is planned to be invited by mail for the focus groups from the population of GPs in the Hamburg metropolitan area and adjacent counties of Lower Saxony and Schleswig-Holstein. The sample will be selected from a register maintained by the Department of General Practice/Primary Care at the University Medical Centre Hamburg-Eppendorf. Heterogeneity in GPs' gender, age, years of professional experience, specialised training in psychosomatic medicine and rural versus urban location of practice are considered possibly relevant variables for differing accounts about handling patients with (S)SD and are thus explicitly welcome. The invitation grants an allowance and credit points by the medical association of Hamburg for vocational training. GPs who participated in the Healthcare Network for Somatoform and Functional Disorders (SOFU-Net) project, a complex interventional study addressing the improvement of SDs' healthcare supply that was simultaneously being conducted in Hamburg, ${ }^{35}$ are excluded to prevent the influence of the programme affecting the results of the current study. At least $10 \%$ of GPs are expected to be willing to participate. ${ }^{36}$

Vignettes describing the history of patients with (S) SD will serve as stimuli for the focus groups. Those are created based on cases from the psychosomatic outpatient department at the University Medical Center Hamburg-Eppendorf. The vignette characters vary in sex, age group and the main type of bodily complaints (pain of different location, functional disturbances in different organ systems and complaints centring on fatigue and exhaustion). Vignettes also vary regarding the ICD-codes that match best with the description given, the number of symptoms and diagnostic barriers that are implicitly embodied in the story. Vignettes are tailored to fulfil each diagnostic criterion of DSM-5's SSD and corresponding DSM-IV diagnoses to a different degree: some vignette characters have medically unexplained symptoms and some have a symptom-related medical disease. Some show excessive illness behaviour, thus fulfilling SSD B-criteria, others do not. The case vignettes offer critical information at specific points in the decision-making process that will be discussed in the focus groups. The case vignettes will be presented and participants will be asked to recount similar experiences and difficulties. Furthermore, they are asked how they relate themselves to these patients and which reactions the GPs expect from them. We want to know what makes the diagnosis of (S)SD difficult in this case and how GPs recognise (S)SD. GPs are asked how they document a tentative diagnosis of (S)SD and how (if at all) they inform their patients about it. Finally, GPs are encouraged to tell their own feelings when facing a patient similar to the vignette character.

Participants will be asked about their strategies in recognising (S)SD and helpful or so far missing tools for diagnosing. Next, the new DSM-5 diagnostic criteria will be introduced to the GPs. Their perceptions of this novelty as well as its impact on routine care will be discussed. Finally, central statements of the clinical practice guideline on 'functional, non-specific and somatoform physical complaints"7 by the German Association of the Scientific Medical Societies will be presented and participants will be asked about their feelings regarding guideline recommendations and about difficulties in realising the key features of the abovementioned guideline.

\section{Part II: Dyadic interviews}

Many studies concentrate on either patients' or GPs' perspective. ${ }^{37}$ Individual interviews with dyads of patients and their treating GPs close this gap and allow for detecting and understanding the needs of both parties and how they perceived the diagnostic process. These 
interviews will depict relationships and dynamics between patients and their GPs and thus help exploring complementary or contrasting perspectives. ${ }^{37}$ Linked interviews seem appropriate to explore ideas for improving healthcare supply. Results from the focus groups will be used to develop interview guidelines for GPs and patients.

GPs will be recruited for interviews as depicted in Part I. GPs who were part of SOFU-Net will be allowed to participate in this part of the study. The participating GPs will be given instructions regarding the required patients' characteristics in order to reduce selection bias. The GP recruits relevant patients of her or his choice whom she or he has seen and to whom an ICD-10 diagnosis of the F45 category or the DSM-5 SSD diagnostic criteria apply. Patients and GPs will be interviewed separately. The interview guideline for these semistructured interviews will be developed by researchers from both institutes based on the results of the content analyses of the focus group discussions. Additionally, participating patients will be interviewed using the relevant parts of the WHO Composite International Diagnostic Interview and the structured clinical interview for DSM disorders to allow for comparison of the cases. We aim for 15 dyadic interviews and perform ongoing qualitative analyses. Depending on the results, another 10 interviewees for 5 dyadic interviews will be recruited. Interviews will last 60-120 min each. GPs and patients will receive an allowance for their interviews.

\section{Part III: Survey}

Based on the content analyses of focus groups and dyadic interviews, a questionnaire will be developed to validate the obtained results in a representative sample of GPs in Germany. The survey will quantify GPs (dis)agreement towards items which comprise the barriers involved in the diagnostic process of (S)SD found to be relevant in the aforementioned parts of this study. GPs will also be asked to rate the clinical utility of both the DSM-IV and DSM-5 conceptualisations of (S) SD. The survey will also evaluate what kind of interventions GPs would prefer or find helpful in order to mitigate diagnostic barriers.

Information on GPs' sociodemographic characteristics such as age, gender, years of professional experience or special training in psychosomatic medicine will be gathered to allow for comparisons of subpopulations of the GPs. A two-phase pretest will be performed with a qualitative part that evaluates comprehensibility and acceptability and a standard, quantitative part. ${ }^{38}$ Afterwards, this questionnaire will be used in a national survey. To conduct a nationwide survey, we will outline our sampling strategy: our target population is GPs who currently work in a private practice in Germany. We aim for a representative sample. To create a sampling frame, we will gather data from online physicians' databases of the German federal states' associations of statutory health insurance physicians and the German federal states' medical associations.

Recruitment of GPs is known to be difficult. ${ }^{39}$ To receive a high response rate, we will send two reminders after 2 and 4 weeks, respectively. Furthermore, the participants will be offered a financial incentive.

\section{Data analysis}

Qualitative data from focus groups and dyadic interviews will be digitally recorded and transcribed verbatim by a trained student research assistant. The transcriptions will be analysed independently by one researcher from the Department of Psychosomatic Medicine and Psychotherapy and one researcher from the Department of Primary Medical Care according to the recommendation of qualitative content analysis. ${ }^{40-42}$ First, both researchers will separately analyse the fragmented passages in the text and then discuss it to reach consensus regarding both the assigned categories and codes. Code categories will be developed using both a conventional strategy, that is, extracting categories directly from the text data and a directed approach, deriving categories from our study groups' review. ${ }^{42}$ To assist the analysis, MaxQDA software will be used. Statistical analysis of quantitative data will be performed with R and SPSS software.

\section{Ethics and dissemination}

The aim of this study is to thoroughly analyse the process that leads to a diagnosis or non-diagnosis of (S) SD in primary care. A mixed method approach is used to explore and weight GPs attitudes and experiences in diagnosing (S)SD. Ethics approval was obtained from the Ethics Committee of the Hamburg Medical Association on 07.04.2015, approval number PV4763. Qualitative research is useful in exploring decision making processes and can provide information for large-scale quantitative studies. ${ }^{43}$ Quantitative research, however, explores the relationships between measurable variables and outcomes. Our qualitative-quantitative approach will allow data to be cross-checked from multiple sources to search for patterns in the data and will give a more detailed and balanced picture of the way (S)SD is dealt with in primary care. This approach will assess the relevance of known barriers, enhancing the reliability of previous studies' findings.

Furthermore, we will investigate GPs self-concept regarding management of (S)SD. Findings will be used to derive strategies and tools to improve diagnosis and management of patients with (S)SD. Since training of GPs has not shown relevant effects on clinical outcomes such as somatisation severity, hypochondriacal fears or physical functioning yet, ${ }^{3} 3031$ other or improved measures have to be identified that fit both GPs' and patients' needs. These measures might address GPs, other medical care providers, patients or other groups engaged. The results of this study will help to shorten the diagnostic delay. This might affect the patients' outcome, the GPs' contentment with the diagnostic process and decreases healthcare costs. ${ }^{324}$

\section{Strengths and limitations}

Healthcare providers other than GPs are not part of this study despite their role in the management of (S)SD. 
Consequently, we do not consider every actor who might hinder or facilitate diagnosing or non-diagnosing of a (S) SD. The methods used do not allow for direct investigation of the diagnostic process; thus, our results are based on other parties' assessment and judgement. Furthermore, participating GPs will not necessarily be aware of the fact that they do not recognise (S)SD. Non-recognition or non-diagnosing of a condition can be due to several factors, including missing knowledge or awareness, unconscious avoidance or an intentional strategy, for example, to save a patient from disincentives or stigmatisation. Thus, we cannot expect getting direct insight into mechanisms that lead to possible non-recognition of (S)SD. The results might reflect aspects being specific for German healthcare providers and the German healthcare system and therefore might possibly not apply for other countries. DSM is a classification system not commonly used in non-psychiatric settings in Germany. The concepts of Bodily (Di-)Stress Disorder/ Syndrome that focus on symptom counts and clusters coexist and might also be relevant, especially in primary care settings, when integrated in the upcoming ICD-11 and ICD-11 Primary Healthcare. ${ }^{44-47}$

The shift in the diagnostic concept of (S)SD has done a $180^{\circ}$ turn over the question of a symptom's explanation which makes comparison with previous findings difficult. ${ }^{49}$ GPs perception of this conceptual change and their assessment of its impact on their daily practice will therefore be investigated in this project. Unlike patients' characteristics that might influence the diagnostic process of $\mathrm{SD},{ }^{16}$ comparatively little is known about physicians' characteristics that might influence the diagnosis. This study will therefore encompass relevant sociodemographic characteristics of a representative sample of German GPs. Compared with depression ${ }^{50}{ }^{51}$ little is yet known about the impact of an early diagnosis of SSD in primary care.

\section{CONCLUSION}

This study will provide methodologically sound knowledge about the diagnostic process and management of (S) SD, its barriers and difficulties in primary care. Investigating the factors that explain current professional practice and any reasons for resisting new practices is the basis for tailoring interventions. Efforts to change professional practice are more likely to succeed when these factors are considered. ${ }^{52}$ Therefore, the results of this study might provide a basis for the development of problem-solving approaches. Especially tailored interventions can help to reduce implementation barriers of evidence based recommendations. ${ }^{53}$ The question of whether adequate treatment of (S)SD will begin earlier if barriers and difficulties that hinder a diagnosis of (S) SD in primary care are better understood and resolved, might be investigated in a follow-up project. Finally, the relation between recognition, diagnosis and outcome has to be addressed in a further study.
The results of this study will be presented to researchers, physicians and other actors through conference talks and publications in relevant journals. The fact that this study will allow for derivation of measures that improve both the patients' outcomes and cost-effectiveness makes it relevant for key stakeholders. To this end, a final report will be published to report the study's results.

Contributors $\mathrm{CH}$ drafted this manuscript. The study's principal investigators BL and MS designed the study with AA and obtained the funding and ethics' approval. NJP, TZ and ML and CH advanced the concept, prepared the study, conduct the study and analyse the data. All authors read and approved the final manuscript.

Funding This study is supported by the Deutsche Forschungsgemeinschaft (DFG); principal investigators are Martin Scherer (SCHE 1689/5-1), Astrid Althaus (AL 1459/5-1) and Bernd Löwe (L0 766/13-1). The project will be conducted, analysed, interpreted and published independently from the funding source.

Competing interests None declared.

Ethics approval Ethics Committee of the Hamburg Medical Association, Germany.

Provenance and peer review Not commissioned; externally peer reviewed.

Open Access This is an Open Access article distributed in accordance with the Creative Commons Attribution Non Commercial (CC BY-NC 4.0) license, which permits others to distribute, remix, adapt, build upon this work non-commercially, and license their derivative works on different terms, provided the original work is properly cited and the use is non-commercial. See: http://creativecommons.org/ licenses/by-nc/4.0/

(c) Article author(s) (or their employer(s) unless otherwise stated in the text of the article) 2017. All rights reserved. No commercial use is permitted unless otherwise expressly granted.

\section{REFERENCES}

1. American Psychiatric Association. Diagnostic and statistical manual of mental disorders. 5th ed. Washington, DC: American Psychiatric Publishing, 2013.

2. American Psychiatric Association. Diagnostic and statistical manual of mental disorders. 4th ed, Text Revision. Washington, DC: American Psychiatric Publishing, 2000.

3. Rief W, Martin A, Rauh E, et al. Evaluation of general practitioners' training: how to manage patients with unexplained physical symptoms. Psychosomatics 2006;47:304-11.

4. Henningsen $P$, Jakobsen $T$, Schiltenwolf $M$, et al. Somatization Revisited. J Nerv Ment Dis 2005;193:85-92.

5. Wiborg JF, Gieseler D, Fabisch AB, et al. Suicidality in primary care patients with somatoform disorders. Psychosom Med 2013;75:800-6.

6. Wiborg JF, Gieseler D, Löwe B. Suicidal ideation in german primary care. Gen Hosp Psychiatry 2013;35:366-9.

7. Schaefert R, Hausteiner-Wiehle C, Häuser W, et al. Non-specific, functional, and somatoform bodily complaints. Dtsch Arztebl Int 2012;109:803-13.

8. Steinbrecher N, Koerber S, Frieser D, et al. The prevalence of medically unexplained symptoms in primary care. Psychosomatics 2011;52:263-71.

9. Roca M, Gili M, Garcia-Garcia M, et al. Prevalence and comorbidity of common mental disorders in primary care. J Affect Disord 2009;119:52-8.

10. Haller $\mathrm{H}$, Cramer $\mathrm{H}$, Lauche $\mathrm{R}$, et al. Somatoform disorders and medically unexplained symptoms in primary care. Dtsch Arztebl Int 2015;112:279-87.

11. Löwe B, Spitzer RL, Williams JB, et al. Depression, anxiety and somatization in primary care: syndrome overlap and functional impairment. Gen Hosp Psychiatry 2008;30:191-9.

12. Schaefert R, Laux G, Kaufmann C, et al. Diagnosing somatisation disorder (P75) in routine general practice using the International classification of primary Care. J Psychosom Res 2010;69:267-77.

13. Warren JW, Clauw DJ. Functional somatic syndromes: sensitivities and specificities of self-reports of physician diagnosis. Psychosom Med 2012;74:891-5.

14. Levenson JL. The somatoform disorders: 6 characters in search of an author. Psychiatr Clin North Am 2011;34:515-24. 
15. Hamilton JC, Eger M, Razzak S, et al. Somatoform, factitious, and related diagnoses in the national hospital discharge survey: addressing the proposed DSM- 5 revision. Psychosomatics 2012;54:142-8.

16. Nimnuan C, Hotopf M, Wessely S. Medically unexplained symptoms: how often and why are they missed? QJM 2000;93:21-8.

17. Kohlmann S, Gierk B, Hilbert A, et al. The overlap of somatic symptoms, anxiety and depression: a Population based analysis. $J$ Psychosom Res 2016;85:68.

18. Hanel G, Henningsen P, Herzog W, et al. Depression, anxiety, and somatoform disorders: vague or distinct categories in primary care? results from a large cross-sectional study. J Psychosom Res 2009;67:189-97.

19. Löwe B, Spitzer RL, Williams JB, et al. Depression, anxiety and somatization in primary care: syndrome overlap and functional impairment. Gen Hosp Psychiatry 2008;30:191-9.

20. Zonneveld LN, Sprangers MA, Kooiman CG, et al. Patients with unexplained physical symptoms have poorer quality of life and higher costs than other patient groups: a cross-sectional study on burden. BMC Health Serv Res 2013;13:520.

21. Alonso J, Angermeyer MC, Bernert S, et al. Disability and quality of life impact of mental disorders in Europe: results from the European Study of the Epidemiology of Mental Disorders (ESEMeD) project. Acta Psychiatr Scand Suppl 2004;109:38.

22. Looper KJ, Kirmayer LJ. Behavioral medicine approaches to somatoform disorders. J Consult Clin Psychol 2002;70:810-27.

23. Hiller W, Fichter MM, Rief W. A controlled treatment study of somatoform disorders including analysis of healthcare utilization and cost-effectiveness. J Psychosom Res 2003;54:369-80.

24. Smith GR, Rost K, Kashner TM. A trial of the effect of a standardized psychiatric consultation on health outcomes and costs in somatizing patients. Arch Gen Psychiatry 1995;52:238-43.

25. Sumathipala $A$. What is the evidence for the efficacy of treatments for somatoform disorders? A critical review of previous intervention studies. Psychosom Med 2007;69:889-900.

26. Kroenke K. Efficacy of treatment for somatoform disorders: a review of randomized controlled trials. Psychosom Med 2007;69:881-8.

27. Zook CJ, Moore FD. High-cost users of medical care. N Engl J Med 1980;302:996-1002.

28. Barsky AJ, Orav EJ, Bates DW. Somatization increases medical utilization and costs independent of psychiatric and medical comorbidity. Arch Gen Psychiatry 2005;62:903-10.

29. Hatcher S, Arroll B. Assessment and management of medically unexplained symptoms. BMJ 2008;336:1124-8.

30. Rosendal $\mathrm{M}$, Olesen F, Fink $\mathrm{P}$, et al. A randomized controlled trial of brief training in the assessment and treatment of somatization in primary care: effects on patient outcome. Gen Hosp Psychiatry 2007;29:364-73.

31. Morriss RK, Gask L. Treatment of patients with somatized mental disorder: effects of reattribution training on outcomes under the direct control of the family doctor. Psychosomatics 2002;43:394-9.

32. Schaefert R, Kirsch G, Kaufmann C, et al. New ways in caring for patients with functional syndromes - interdisciplinary group intervention in the primary care setting: a qualitative study. Psychother Psychosom Med Psychol 2013;63:167.

33. van der Feltz-Cornelis $\mathrm{CM}$, van Oppen $\mathrm{P}$, Adèr $\mathrm{HJ}$, et al. Randomised controlled trial of a collaborative care model with psychiatric consultation for persistent medically unexplained symptoms in general practice. Psychother Psychosom 2006;75:282-9.
34. Murray AM, Toussaint A, Althaus A, et al. The challenge of diagnosing non-specific, functional, and somatoform disorders: a systematic review of barriers to diagnosis in primary care. $J$ Psychosom Res 2016;80:1-10.

35. Shedden-Mora MC, Gross B, Lau K, et al. Collaborative stepped care for somatoform disorders: a pre-post-intervention study in primary care. J Psychosom Res 2016;80:23-30.

36. Thorpe C, Ryan B, McLean SL, et al. How to obtain excellent response rates when surveying physicians. Fam Pract 2008;26:65-8.

37. Kendall M, Murray SA, Carduff E, et al. Use of multiperspective qualitative interviews to understand patients' and carers' beliefs, experiences, and needs. BMJ 2009;339:b4122.

38. Prüfer M. Kognitive interviews. Mannheim: Zentrum für Umfragen, Methoden und Analysen, 2005. http://nbn-resolving.de/urn:nbn:de: 0168-ssoar-201470.

39. Johnston S, Liddy C, Hogg W, et al. Barriers and facilitators to recruitment of physicians and practices for primary care health services research at one centre. BMC Med Res Methodol 2010;10:109.

40. Mayring P. Qualitative inhaltsanalyse. 6th ed. Weinheim: Deutscher Studien Verlag, 1997.

41. Schreier M. Ways of doing qualitative content analysis: disentangling terms and terminologies. Forum: Qualitative Social Research 2014;15:1 http://nbn-resolving.de/urn:nbn:de:0114-fqs1401185

42. Hsieh HF, Shannon SE. Three approaches to qualitative content analysis. Qual Health Res 2005;15:1277-88.

43. Britten N. Qualitative interviews in medical research. BMJ 1995;311:251-3.

44. Fink P, Toft T, Hansen MS, et al. Symptoms and syndromes of bodily distress: an exploratory study of 978 internal medical, neurological, and primary care patients. Psychosom Med 2007;69:30-9.

45. Goldberg DP, Reed GM, Robles R, et al. Multiple somatic symptoms in primary care: a field study for ICD-11 PHC, WHO's revised classification of mental disorders in primary care settings. $J$ Psychosom Res 2016;91:48-54

46. Lam TP, Goldberg DP, Dowell AC, et al. Proposed new diagnoses of anxious depression and bodily stress syndrome in ICD-11-PHC: an international focus group study. Fam Pract 2013;30:76-87.

47. ICD-11 beta draft. apps.who.int. http://apps.who.int/classifications/ icd11/browse/f/en\#!/http\%3A\%2F\%2Fid.who.int\%2Ficd\%2Fentity\% 2F767044268 (accessed 3 May 2017).

48. Voigt K, Wollburg E, Weinmann N, et al. Predictive validity and clinical utility of DSM-5 Somatic Symptom Disorder: prospective 1-year follow-up study. J Psychosom Res 2013;75:358-61.

49. Wollburg E, Voigt K, Braukhaus C, et al. Construct validity and descriptive validity of somatoform disorders in light of proposed changes for the DSM-5. J Psychosom Res 2013;74:18-24.

50. Goldberg D, Privett M, Ustun B, et al. The effects of detection and treatment on the outcome of major depression in primary care: a naturalistic study in 15 cities. Br J Gen Pract 1998;48:1840-4.

51. Ormel J, Van Den Brink W, Koeter MW, et al. Recognition, management and outcome of psychological disorders in primary care: a naturalistic follow-up study. Psychol Med 1990;20:909-23.

52. Baker R, Camosso-Stefinovic J, Gillies C, et al. Tailored interventions to address determinants of practice. Cochrane Database Syst Rev 2015:CD005470.

53. Sinnema $\mathrm{H}$, Terluin $\mathrm{B}$, Wensing $\mathrm{M}$, et al. Systematic tailoring for the implementation of guideline recommendations for anxiety and depressive disorders in general practice: perceived usefulness of tailored interventions. BMC Fam Pract 2013;14:94. 